\title{
LEAD IN DUST AND SOIL FROM DAY-CARE CENTRES
}

Brian Gulson, Professorial Fellow (Macquarie University) / Chief Research Scientist (CSIRO)

James Ray, Graduate student, Macquarie University

ead is a neurotoxin and, even at low levels in the blood, is Lconsidered to result in lowering of IQ, behavioural problems and learning difficulties in children ${ }^{1}$.

More than 90 per cent of the lead in Sydney urban air is still derived from motor vehicle emissions $s^{2}$. With many schools and day-care centres located on major traffic thoroughfares, and often with traffic lights located outside the schools for pedestrian safety, there is a potential risk of rooms and play areas becoming contaminated with lead. The main pathways of petrol lead to children are via direct airborne transport from the roads, or indirectly by re-entrainment of dust/soil by the wind. This airborne lead may be deposited onto surfaces such as desks, floors, benches and paved areas, and hence ingested via hand-to-mouth activity.

Airborne lead may also be deposited in ceiling cavities and, if disturbed, result in contamination of surrounding areas. Contamination from ceiling dust may also occur in older buildings in which ceiling rosettes or cornices are in poor condition. In addition to petrol, lead and other toxic metals may also be introduced into ceiling cavities (and other areas) from previous industrial activities including incinerators and power stations.

Of even greater concern in older areas of inner Sydney is the contribution from leaded paint, with any dwelling built before 1970 having the potential to contain lead-bearing paints $^{3}$. If these paints are in poor condition or the dwelling is being renovated, there is a chance to contaminate not only the immediate dwelling but also the surrounding neighbourhood ${ }^{4}$. Such contamination can result in elevated blood lead levels in children and even in adults.

Because many day-care centres are in older buildings and in areas of moderate traffic, we undertook a pilot investigation to determine the extent of lead contamination in several day-care centres, especially within the inner Sydney area.

\section{METHODS}

Six day-care centres - five from inner Sydney suburbs agreed to participate in the study. These centres were in relatively old buildings with the possibility of lead paints (and lead from other sources) being present.

A variety of samples was taken at each of the centres depending on the nature of ambient lead sources (internal and external) and their accessibility, especially to children. The interior areas targeted for identification of lead dust contamination included windowsills, shelves and carpeted floors. Potentially contaminated surfaces were sampled using commercially available moistened wipes such as Diaparene Baby Wash Cloths. Dust from carpets and rugs was collected over a known surface area using a Volta vacuum cleaner dedicated to such sampling. Long-term dust (passive dustfall accumulation) was collected using petri dishes ${ }^{5}$ placed in specific locations for two to four months.

Exterior samples were taken of surface soil in playground areas and from the sandpit or from areas potentially contaminated by lead paint such as under the eaves. Interior and exterior samples of paint flakes were taken from any painted surface which was in poor condition, especially in areas which were accessible to children.
Further details of sampling and analytical methods, and complete analyses, may be obtained from the first author.

\section{RESULTS}

Results for soil and dust are displayed in Figures 1,2 and 3. The results of this study can be compared with "guidelines" provided by regulatory authorities. For example, the "action level" of the NSW Environment Protection Authority in soils is $300 \mu \mathrm{g} / \mathrm{g} \mathrm{Pb}$ (ppm or $\mathrm{mg} / \mathrm{kg}$ ). No guidelines are available for dustfall accumulation (petri dish dust) but the US Department of Housing and Urban Development ${ }^{6}$ requires that the "clean-up" levels for lead loading (amount of $\mathrm{Pb}$ per unit area) using dust from surface wipes on floors after lead abatement is $1,076 \mu \mathrm{g} / \mathrm{m}^{2}$. Likewise, there are no guidelines for vacuum cleaner dust, although Bawden-Smith et al. ${ }^{7}$ give values of $500-1,000 \mu \mathrm{g} / \mathrm{g}$.

Day-Care Centre A was a new building (built in 1987) containing no obvious internal lead sources and was located away from any main roads. The levels found in the playground soil and the sandpit were considered equivalent to "background" levels (Figure 1).

Day-Care Centre B was also in a low traffic zone, and again the sandpit and soil lead levels were very low (Figure 1). The building itself was old, though the interior had recently been repainted and recarpeted so the vacuum dust, petri dish dust and surface wipe lead levels were also low.

Day-Care Centre $\mathbf{C}$ was in an older suburb of Sydney in a low traffic zone. The playground soil and sandpit lead levels were again low. In the older section of the centre, there were high ceilings with rosettes which may have allowed dust entry. As surfaces were regularly cleaned, the lead levels in the dust wipes were generally low. However, dust accumulation for longer periods was higher as measured in the vacuum cleaner dust, a music room surface wipe (taken along a high ledge), and the dustfall accumulation over a 30-day period from the petri dish dust.

Day-Care Centre $\mathbf{D}$ proved to be the most contaminated day-care centre in this study. Paint under the eaves was in poor condition and contained about 45 per cent lead. Deterioration of this paint contaminated the soil beneath the eaves and probably the playground soil. The sandpit sand had recently been replaced, resulting in the negligible lead levels present.

Some interior paint surfaces were found to contain up to 16 per cent $\mathrm{Pb}$. This paint, however, was in relatively good condition. Surface wipes from along windowsills in two rooms contained high lead values of $1,020 \mu \mathrm{g} \mathrm{Pb} / \mathrm{m}^{2}$ and $5,310 \mu \mathrm{g} \mathrm{Pb} / \mathrm{m}^{2}$. These windows were below the eaves with deteriorating lead paint and as the windows were opened during the day, lead-bearing dust may have been blown in.

Even though most rooms contained ventilators and one room had poorly fitted "tongue and groove" ceiling panelling, the amount of dust entering the rooms through these pathways was low as shown by the low amounts of lead in the three-month dustfall accumulation. The baby room was in excellent condition and, being a more recent addition to the building and away from the eaves with deteriorating paint, contained very low lead levels.

Day-Care Centre $\mathbf{E}$ was in an area of moderate traffic in an older suburb of Sydney. The day-care centre section of the building was relatively new and in good condition. The lead levels in all samples were low. 


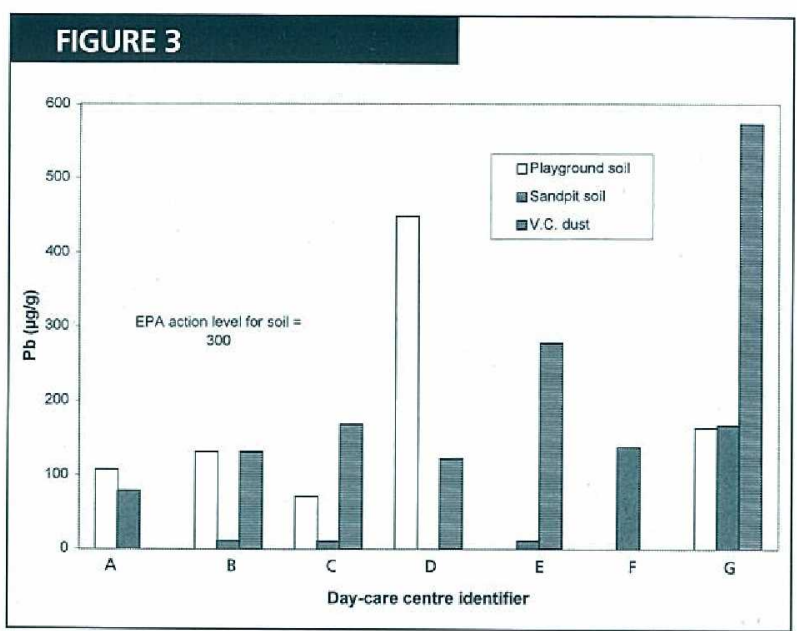

Lead concentrations in soil and dust from day-care centres in Sydney. A blank space indicates that no sample was available. The soil sample from Centre $\mathrm{D}$ containing $2,090 \mathrm{ppm} \mathrm{Pb}$ is not plotted. The action level for contaminated soils designated by the NSW EPA is shown. The vacuum cleaner dust results can be compared with a mean value of $2,255 \mathrm{w} 4,783 \mathrm{ppm} \mathrm{Pb}$ (median 1,000 ppm) found in 22 houses from inner Sydneys.

\section{FIGURE 4}

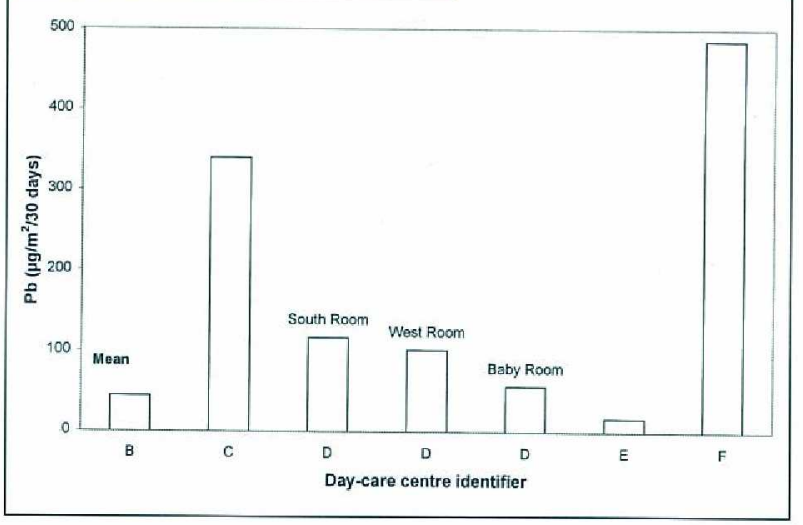

Comparison of lead in dustfall accumulation (petri dust) representing airborne dust deposited over a designated time interval. The mean and standard deviation $(90 \pm 60)$ for 164 samples from other Sydney houses is taken from Gulson et al. ${ }^{9}$.

\section{FIGURE 5}

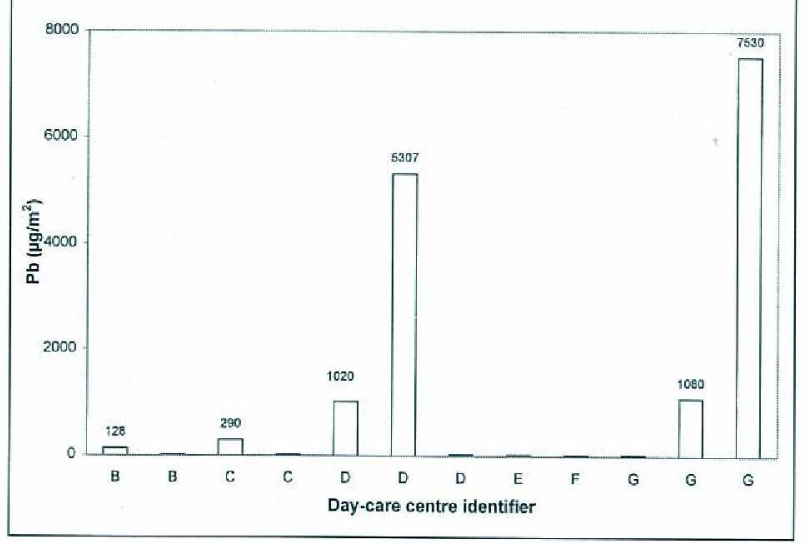

Lead concentrations in surface wipes from the day-care centres. Where no value is given, the result is $<10 \mu \mathrm{g} \mathrm{Pb} / \mathrm{m}^{2}$. The level of clean-up after lead paint remediation recommended by the US Department of Housing and Urban Development is $1,076 \mu \mathrm{g} / \mathrm{m}^{2}$.
Day-Care Centre $\mathbf{F}$ was in a high traffic area and this may be the explanation for the slightly elevated sandpit lead levels. The interior was in good condition and had no carpeted areas.

Day-Care Centre $\mathbf{G}$ was also in a high traffic area and this may be the explanation for the elevated lead levels in the playground soil and sandpit. Inside, there was a high, exposed, old timber ceiling. After high winds the staff noted that black dust could be seen on surfaces. With regular cleaning the lead levels are kept very low, as shown by the vacuum dust and surface wipe (cupboard) results. However, high lead levels can accumulate over time as evidenced from the wipes of surfaces not cleaned regularly and from the dustfall accumulation over an 80-day period in a petri dish (490 $\mu \mathrm{g} \mathrm{Pb} / \mathrm{m}^{2}$ per 30 days).

\section{DISCUSSION}

Results from this pilot study were encouraging in that most measured levels were below any guideline level. Only one centre with deteriorating lead paint posed a hazard to the children, carers and the neighbourhood. Since the results of the study were provided to this centre, remedial actions have been implemented. However, there is a clear need for public health authorities, in conjunction with the Environment Protection Authority, to develop guidelines for interior sampling methods and clean-up levels.

The levels of lead in soil and dust measured in this pilot study of day-care centres varied considerably depending on factors which include location, and age and condition of the building. In general, centres housed in relatively new buildings have minimal problems unless they are near major traffic thoroughfares. When near such areas, exterior sites such as the sandpit and play areas may become contaminated over time. These problems have been minimised by regular replacement of the sand, covering of the sandpit when not in use, and installation of paving so daily hosing is possible.

In older buildings the potential hazards are paint and ceiling dust, typified by centres D and G respectively in this study. Remediation of leaded paint can be expensive, as can be ceiling dust from high, panelled ceilings commonly found in day-care centres housed in old church buildings.

On the positive side, regular cleaning by the carers of the day-care centres investigated in this pilot study has minimised the risk to the young children even in centres which are potentially a major hazard. Regular cleaning encompasses surface wiping with moist cloths and vacuuming of carpets and mats. Given the low levels of lead in all except one centre, it was not considered necessary to inconvenience the children, carers or parents with the second stage of this study, i.e. blood sampling. This does not mean the children have low blood lead levels, as they may live in a neighbourhood or dwelling where renovation and contamination from old leaded paint are ubiquitous.

This is the first published study that we know of specifically directed towards environmental sampling of day-care centres in Australia, so comparisons are not possible. With changes in Federal Government funding to day-care centres, extra attention to this area from public health authorities may be essential to ensure the health and safety of our infants. 


\section{Lead in dust and soil from day-care centres}

\section{Continued from page 95}

\section{ACKNOWLEDGMENTS}

We thank: Macquarie University for financial support for this study under the Macquarie University Research Grants Scheme; Ms Ginie Udy of the Uniting Church Children's Services Forum for access to the day-care centres; the coordinators of the centres for their assistance; Professor Barry Batts for access to the ICP-AES; and Graeme Waller for the surface wipe digestion method.

1. National Center for Lead Safe Housing, Maryland. Guidelines for the evaluation of lead-based paint hazards in housing. February 1995. 2. Bawden-Smith J, McAnulty J, Bek M. Lead exposure at a school for children with developmental disabilities. NSW Public Health Bulletin 1995; 6(11):124-6.

3. Fett MJ, Mira M, Smith J et al. Community prevalence survey of children's blood lead levels and environmental lead contamination in inner Sydney. Med J Aust 1992; 157:441-5.

4. Gulson BL, Jameson CW, Mahaffey KR, Mizon KJ, Korsch MJ, Vimpani G. Pregnancy increases mobilisation of lead from maternal skeleton. J Lab Clin Med 1997; 130:51-62.

5. National Research Council. Measuring lead exposure in infants, children, and other sensitive populations. Washington DC: National Academy Press, 1993.

6. Chiaradia M, Gulson BL, Mizon KJ, James M, Jameson CW, Johnson D. Identification of secondary lead sources in the air of an urban environment. Atmos Environ 1997; 31:3511-21.

7. NSW Government Lead Issues Paper. Sydney: Environment

Protection Authority, 1993.

8. Gulson BL, Davis JJ, Bawden-Smith J. Paint as a source of recontamination of houses in urban environments and its role in maintaining elevated blood leads in children. Sci Total Environ 1995 maintainin

164:221-35.
9. Gulson BL, Davis JJ, Mizon KJ, Korsch MJ, Bawden-Smith J. Sources of lead in soil and dust and the use of dust fallout as a sampling medium. Sci Total Environ 1995; 166:245-62.

\section{EDITORIAL NOTE}

Lead is a ubiquitous contaminant of the urban environment. Children are at greatest risk of exposure and harm from absorption of environmental lead by virtue of their behaviour, their metabolism and the sensitivity of the developing nervous system to the toxic effects of lead. Lead in paint and lead additives in fuel are two sources of lead exposure in urban areas. This study confirms that lead is readily detected in the home and play environment of many children in NSW. A recent study showed that average blood lead levels in NSW preschool children were 5 to $7 \mu \mathrm{g} / \mathrm{dL}$, with less than 25 per cent of inner-city children being above the goal for blood lead of $10 \mu \mathrm{g} / \mathrm{dL}^{1}$. This is an improvement since $1992^{2}$. Reductions in the proportion of cars using leaded fuel and in the the lead added to that fuel have reduced exposure. A study in Sydney showed that traffic flow near a child's home was a more important determinant of blood lead level than the traffic flow at the child's child-care centre ${ }^{3}$. These results do not detract from the need for efforts to reduce exposure to environmental lead, such as those now being coordinated by the Lead Reference Centre.

1. Mira M, Bawden-Smith J, Causer J, et al. Blood lead concentrations of preschool children in Central and Southern Sydney. Med J Aust 1996; 164:399-402.

2. Fett M, Mira M, Smith J, et al. Community prevalence survey of children's blood lead levels and environmental lead contamination in inner Sydney. Med J Aust 1992; 157:441-445.

3. Cowie C, Black D, Fraser I. Blood lead levels in preschool children in eastern Sydney. Aust NZ J Public Health 1997; 21:755-760.
Measuring the amount of lead in indoor dust

\section{Continued from page 93}

Thus, the petri dish method can be used as a measure of recontamination, allowing individuals and health authorities to assess the effectiveness of abatement strategies.

Although no single method has yet been established as a standard for measuring indoor lead exposure, the long-term dust-fall accumulation method has advantages: it has low cost, does not require a power source, causes minimal inconvenience to the householder, does not involve noise, can be measured over a specific period, and cannot be biased by the householder cleaning the house before a sample visit ${ }^{4}$

The disadvantages of the method are the potential for disturbance or contamination and the time delay to obtain a measurement. In our study only one dish was lost and there were no overt signs of contamination.

Further evaluation of the technique to quantify the direct relationship between lead loading and blood lead levels is under way in North Lake Macquarie and Broken Hill. Our pilot study has shown that the method provides a valuable indicator of comparative trends in lead exposure over time and between areas.

1. Royal Melbourne Institute of Technology. Reducing Lead Exposure in Australia: Risk Assessment and Analysis of Economic, Social and Environmental Impacts. Canberra: Australian Government Publishing Environmental Impacts. Canberra. Australian Government Publishin Service, 199

2. Rinehart R, Rogers J, Vendetti M. Wipe/vacuum lead in house dust sampling, draft report to US EPA, 1993.

3. Jacobs DE. Guidelines for evaluation and control of lead paint hazards in housing. The National Centre for Lead-Safe Housing for US Department of Housing and Urban Development, 1994

4. Gulson BL, Davis JJ, Mizon KJ, Korsch MJ, Bawden-Smith J. Sources of lead in soil and dust and the use of dust fallout as a sampling medium. Submitted to Sci Total Eviron 1995; 166:245-262.

\section{Update on lead-related activities}

\section{Continued from page 99}

In June 1997, \$4.6 million was received from the NSW Health Department, the NSW Environment Protection Authority and Pasminco Metals-Sulphide for ongoing remediation.

\section{NORTHERN SYDNEY AREA PUBLIC HEALTH UNIT}

Local Government Environmental Health Officers were invited to a regional workshop on roles and responsibilities for lead hazard management conducted by the Lead Reference Centre and the Northern Sydney Area Public Health Unit on 9 December 1997 at Macquarie Hospital, North Ryde. Representatives from local authorities within the area, NSW Environment Protection Authority, WorkCover and Environmental Health Officers from the NSAHS Public Health Unit attended.

Documents distributed included Local Government Lead Management and Guidelines for Environmental Health Officers in managing cases of Elevated Blood Lead Levels. The aim of of the workshop was to provide information about lead hazards and the powers available to deal with lead problems. The outcomes of the day were an awareness of the responsibility of local government in the approval of building applications, procedures to be recommended for building renovations and case investigation protocol. 